\title{
Effects of Lactobacillus plantarum and Leuconostoc mesenteroides Probiotics on Human Seasonal and Avian Influenza Viruses
}

\author{
Joon-Yong Bae ${ }^{1 \dagger}$, Jin Il Kim ${ }^{1 \dagger}$, Sehee Park ${ }^{1}$, Kirim Yoo ${ }^{1}$, In-Ho Kim² ${ }^{2}$, Wooha Joo ${ }^{3}$, Byung Hee Ryu ${ }^{3}$, Mee Sook Park ${ }^{1}$, \\ Ilseob Lee ${ }^{1 *}$, and Man-Seong Park ${ }^{1 *}$ \\ ${ }^{1}$ Department of Microbiology, Institute for Viral Diseases, College of Medicine, Korea University, Seoul 02841, Republic of Korea \\ ${ }^{2}$ Korea Food Research Institute, Wanju 55365, Republic of Korea \\ ${ }^{3}$ Daesang Co., Ltd., Icheon 17384, Republic of Korea
}

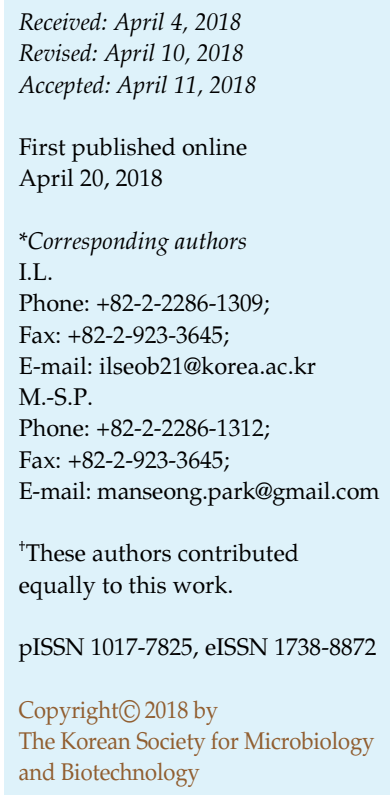

Influenza viruses that cause recurrent seasonal epidemics to humans can be controlled with vaccine and antiviral therapy. However, the medical treatments often exhibit limited efficacy in the elderly or immunosuppressed individuals. In these cases, daily uptake of probiotics may be an option to bring in health benefits against influenza. Here, we demonstrate the effects of probiotics Lactobacillus plantarum (Lp) and Leuconostoc mesenteroides (Lm) against seasonal and avian influenza viruses. As assessed by the plaque size reduction of human $\mathrm{H} 1 \mathrm{~N} 1$ and avian influenza H7N9 viruses, including green fluorescent protein-tagged H1N1 strain in cells, the selected Lp and Lm strains restrained viral replication in mouse lungs with statistical significance. Against lethal viral challenge, the Lp and Lm strains exhibited their beneficial effects by increasing the mean days and rates of survival of the infected mice. These results suggest that, despite rather narrow ranges of protective efficacy, the dietary supplement of Lactobacillus and Leuconostoc probiotics may promote health benefits against influenza.

Keywords: Dietary supplement, influenza, Lactobacillus plantarum, Leuconostoc mesenteroides, probiotic

\section{Introduction}

Recurrent seasonal epidemic and sporadic pandemic influenzas have caused severe public health burdens [1, 2]. Vaccination is considered as the best preventive measure. However, there are often mismatches between the vaccine virus and circulating strains of the season. Furthermore, owing to the constant presence of the virus among aquatic birds as its reservoir [3], zoonotic transmission from animals to humans, as shown in recent outbreaks of H7N9 [4], cannot be prevented effectively. Vaccine mismatch is due to the constantly changing antigenicity of the virus, termed "antigenic drift." Zoonotic transmission of the virus introduces to humans completely different influenza virus strains, which is termed "antigenic shift" [5]. Antigenic drifts and shifts can be prepared against theoretically by a "universal" vaccine [6], which is yet to come. In the meantime, the empirical "word of mouth" preventive measures to reduce susceptibility to the virus or to have reduced illnesses from influenza have come under scientific scrutiny.

Many lactic acid bacteria (LAB) have been recognized as probiotics. LAB are a group of bacteria belonging to many genera, including Lactobacillus and Leuconostoc. Reported health benefits of LAB from the uptake of fermented food are numerous, ranging from the reduction of gut symptoms and diabetes to antitumor effects and enhanced defenses against infectious diseases through immune modulation. The versatility of LAB might be attributed to the diversity of the metabolic products of different probiotic LAB strains $[7,8]$.

The effects of LAB on respiratory tract infections have also been studied actively. In a double-blind, randomized 
controlled trial in the elderly, dietary consumption of LAB was reported to reduce the duration and severity but not the incidence of common cold episodes [9]. Similarly, reduction of the duration of respiratory infections in the elderly by consumption of fermented dairy products containing the probiotic Lactobacillus was observed in a randomized controlled trial [10]. Immune enhancement effects of LAB could have been one of the potential mechanisms explaining such results. However, human trials have not shown definitively the immune enhancement effects of LAB. Boge et al. [11] reported that daily consumption of fermented dairy drink containing Lactobacillus casei DN-114 001 improved antibody responses to influenza vaccination in the elderly in two randomized controlled trials. On the other hand, in a different double-blind, randomized placebo-controlled trial with healthy elderly nursing home residents, daily consumption of fermented dairy drink containing Lactobacillus casei Shirota showed no statistically or clinically significant effects on the protection against respiratory symptoms and no significant differences regarding the influenza-vaccination immune response [12]. Interestingly, the authors of the latter report cautiously pointed out that there was a nonsignificant trend with relative risk reduction whereas most studies often demonstrated beneficial effects that are often difficult to reproduce [8]. Different cocktails of bioactive molecules and metabolites that were released from and by the activity of different strains of LAB could have produced non-uniform outcomes [13].

Kimchi is a traditional Korean food of fermented vegetables. Kimchi is known for its rich source of LAB [14, 15], and LAB isolated from kimchi have manifested many beneficial effects in experiments using animal models [16, 17]. It was also recognized that different LAB strains isolated from different types of kimchi show different immune modulatory functions [18].

The protective effects of LAB isolated from kimchi have been studied against influenza [19]. We also reported antiinfluenza effects of an oral uptake of heat-killed kimchiisolated LAB, Lactobacillus plantarum ( $\mathrm{nF} 1)$, in H1N1 and H3N2 influenza virus-infected mice [17]. As with many other studies looking for anti-influenza effects of LAB using different heat-killed LAB strains and feeding regimens in mice [20,21], we could see a "trend" of protective effects of $L A B$ against influenza. It appears that the efforts to identify a specific strain of LAB that is definitely effective against the virus need to be continued with diverse approaches. One might also take note of the differences of responses between using live and heat-killed LAB [22]. In this study, we determined the effects of a panel of Lactobacillus plantarum and Leuconostoc mesenteroides strains, originally isolated from kimchi, against $\mathrm{H} 1 \mathrm{~N} 1$ and H7N9 influenza viruses in vitro and in vivo.

\section{Materials and Methods}

\section{Ethics}

The animal experiments were conducted in strict accordance with the recommendations delineated in the Guide for the Care and Use of Laboratory Animals of the Animal, Plant and Fisheries Quarantine and Inspection Agency of Korea. The experimental protocols were approved by the Institutional Animal Care and Use Committee (IACUC) of Korea University College of Medicine (Permit No. KOREA-2016-0113).

\section{Viruses and Cells}

A/Korea/01/2009 (2009 pandemic influenza A H1N1 virus, rK09; provided by Korean Centers for Disease Control and Prevention, Korea) [23, 24], K09 harboring serine residues at neuraminidase (NA) residues 58-59 (rK09/NA:NN58SS) [25], A/ Puerto Rico/8/1934 expressing green fluorescent protein (rPR8GFP; provided by Dr. Adolfo Garcia-Sastre, Icahn School of Medicine at Mount Sinai, USA) [26, 27], and A/Anhui/01/2013 (avian influenza A H7N9 subtype 6:2 vaccine virus, rAH01; previously generated by Lee et al. [28]) were propagated in 10-dayold embryonated chicken eggs. Virus titers were determined by a standard plaque assay in Madin-Darby canine kidney (MDCK) cells, which were obtained from the American Type Culture Collection (ATCC, USA). The MDCK cells were maintained in Eagle's Minimum Essential Medium (Lonza, Switzerland) supplemented with $10 \%$ fetal bovine serum (BioTechnics Research Inc., USA) and $1 \%$ penicillin and streptomycin (Gibco, USA) at $37^{\circ} \mathrm{C}$ in a $5 \% \mathrm{CO}_{2}$ incubator. Genomic sequences of all the viruses were confirmed using reverse-transcription PCR before use.

\section{Probiotics Preparation}

Lactobacillus plantarum (Lp; strains J1-8, 330, CK10, and 920; Patent Application Publication No. Korea/10-1249824, 10-1485182, 10-10-1287120, and 10-1600940, respectively) and Leuconostoc mesenteroides (Lm; strains DRC1506 and 218; Patent Application Publication No. Korea/10-1809447 and 10-1055949, respectively) were provided by Daesang Corporation (Korea). Bacterial culture of Lp and Lm strains was performed using De Man-RogosaSharpe (MRS) broth media (MB Cell, USA) at $37^{\circ} \mathrm{C}$. The cultured bacteria were washed with phosphate-buffered saline (PBS; Lonza, USA) by repeating centrifugation $\left(3,500 \times g, 37^{\circ} \mathrm{C}, 20 \mathrm{~min}\right)$ and finally resuspended with PBS again. OD values of the bacteria were measured, and their colony-forming unit (CFU) titers were determined with serial 10 -fold dilutions (down to $10^{-8}$ ) by 
counting the number of colonies grown in MRS agar plates (MB Cell, USA).

\section{Cell-Based Assays}

A virucidal assay was performed by modifying a protocol described previously [29]. Briefly, 100 plaque-forming units (PFU) of a virus (in $100 \mu \mathrm{l}$ PBS; rK09 and rAH01) were mixed with the same volume of Lp and Lm strains (2-fold dilutions from $10^{8} \mathrm{CFU}$ ) and incubated at $37^{\circ} \mathrm{C}$ for $1 \mathrm{~h}$. A standard plaque assay was then performed to determine viral plaque formation in MDCK cells [28]. For the plaque-reduction assay, MDCK cells were infected with 100 PFU of a virus (in $100 \mu \mathrm{l}$ PBS; rK09, rAH01, and rPR8GFP) for $1 \mathrm{~h}$. After being washed three times with cell culture media, the cells were overlaid with Oxoid agar (Thermo Fisher Scientific, USA) containing 2-fold dilutions of $10^{8}$ CFU Lp or Lm strain. The virus-infected, agar-overlaid cells were then stained with crystal violet at $48-72 \mathrm{~h}$ post-infection (hpi). For the detection of GFP expression from the cells infected with rPR8-GFP, each viral plaque was assessed using the EVOS FL Imaging System (Thermo Fisher Scientific, USA) from low to high magnification at 48-72 hpi. PBS was used for the mock treatment.

\section{Mouse Experiment}

Twelve BALB/c mice (5-week-old females; NaraBiotech, Korea) per group were acclimated for 1 week before the start of the experiment. The mice were fed once daily with Lp or Lm strain (10 $0^{9}$ CFU in $200 \mu \mathrm{l}$ PBS) using a feeding needle (model JD-S-124; Jeung Do B\&P, Korea) for 14 days prior to and 14 days after lethal viral challenge with rK09/NA:NN58SS $(2 \times 50 \%$ mouse lethal dose; $2 \mathrm{MLD}_{50}$ ). At 3 and 6 days post-infection (dpi), three mice per group were sacrificed for lung collection, which was used for the titration of viral replication by the plaque assay. Body weight changes and survival rates of the rest of the six mice per group were monitored daily for $14 \mathrm{dpi}$. Mice that lost greater than $25 \%$ of their initial body weight were considered dead and euthanized humanely. PBS was used for the mock infection and treatment as controls.

\section{Statistical Analysis}

Differences in viral lung replication were tested for significance using Student's $t$-test $\left({ }^{*} p<0.05\right)$. Differences in mouse survival rates were tested for significance using the Kaplan-Meier test $(p<0.05)$.

\section{B}
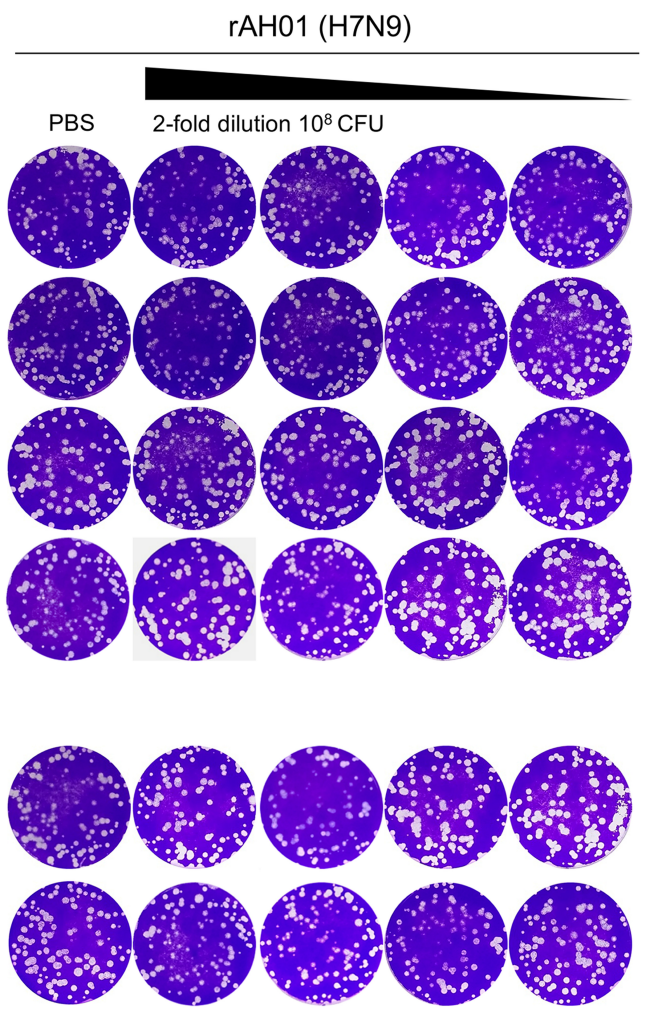

Fig. 1. Virucidal efficacy of Lactobacillus plantarum (Lp) and Leuconostoc mesenteroides (Lm) probiotics against 2009 pandemic influenza H1N1 and avian influenza H7N9 viruses.

Four Lp (J1-8, 330, CK10, and 920) and two Lm (DRC1506 and 218) strains were evaluated for their virucidal efficacy against rK09 (A, 2009 pandemic H1N1 virus) and rAH01 (B, human-infecting avian influenza H7N9 vaccine virus) in MDCK cells. Serial 2-fold dilutions of $10^{8}$ CFU of each probiotic were used in the assay, and PBS was used as a negative control. 


\section{Results}

Lactobacillus plantarum and Leuconostoc mesenteroides Probiotics Restrain Plaque Formation of Human Seasonal H1N1 and Avian Influenza H7N9 Viruses in MDCK Cells

Four strains (J1-8, 330, CK10, and 920) of Lp and two strains (DRC1506 and 218) of Lm were evaluated for their anti-influenza activity in cell-based assays. In the virucidal assay, which might examine the direct efficacy of the Lp and Lm strains, the probiotics exhibited no effectiveness against both rK09 and rAH01. Neither plaque number nor diameter were affected, compared with the PBS-treated wells (Fig. 1). As seen in the virucidal assay, Lp J1-8 and Lm DRC1506 did not demonstrate their effectiveness in the plaque-reduction assay, either. However, treatment with Lp 330, CK10, and 920 and Lm 218 reduced the sizes of viral plaques of rK09 and rAH01 in a dose-dependent manner, even though the plaque numbers appeared to be unaffected (Fig. 2).

To further validate the effects of Lp and Lm probiotics, we used rPR8-GFP in the same plaque-reduction assay. In fluorescent microscopy, we observed that viral plaques of PBS-treated controls expressed green fluorescence. However, treatment with Lp 330, CK10, and 920 and Lm 218 reduced fluorescence expression in a dose-dependent manner, whereas Lp J1-8 and Lm DRC1506 exhibited no effects, either (Fig. 3), as demonstrated against both rK09 and rAH01 (Fig. 2). In particular, at $10^{8} \mathrm{CFU}$ treatment with Lp 330, CK10, and 920 and Lm 218, it was apparent that viral plaques were extremely reduced in diameter (Fig. 3). These results demonstrate the protective effects of Lp 330, CK10, and 920 and Lm 218 against both human and avian influenza viruses.

Treatment with Lactobacillus plantarum and Leuconostoc mesenteroides Probiotics Promotes Survival of Mice Infected with Influenza Virus

We then investigated the protective effects of the Lp 330, CK10, and 920 and Lm 218 strains against lethal viral challenge in mice (Fig. 4A). Given the viral titers determined
A
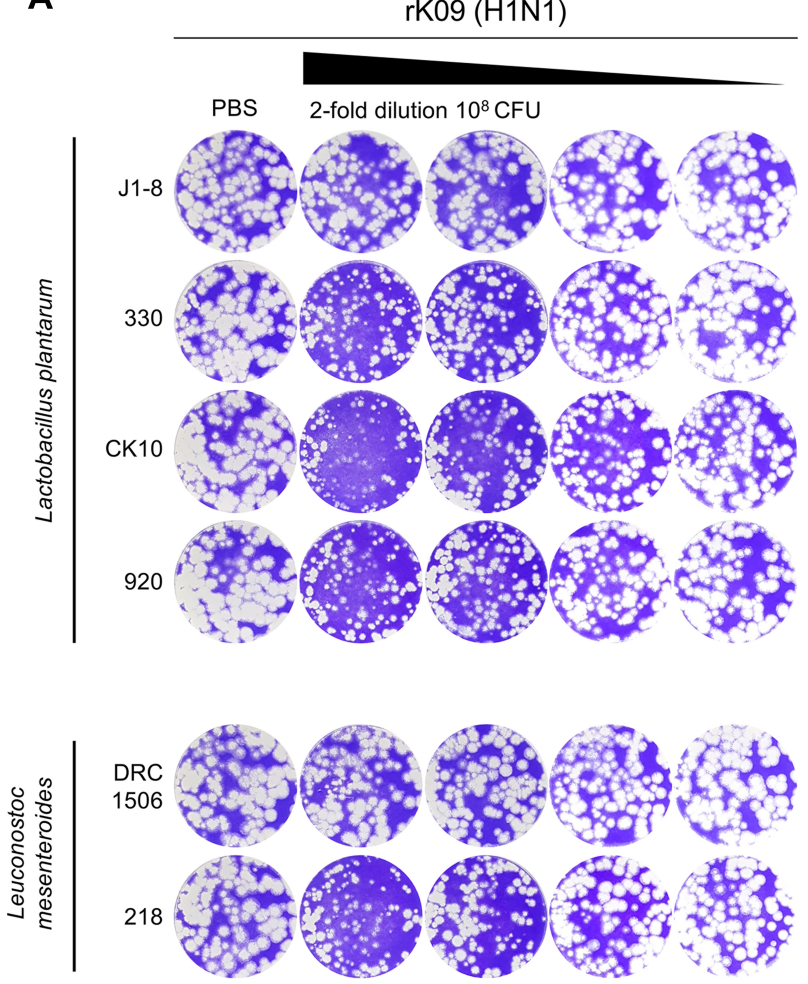

B
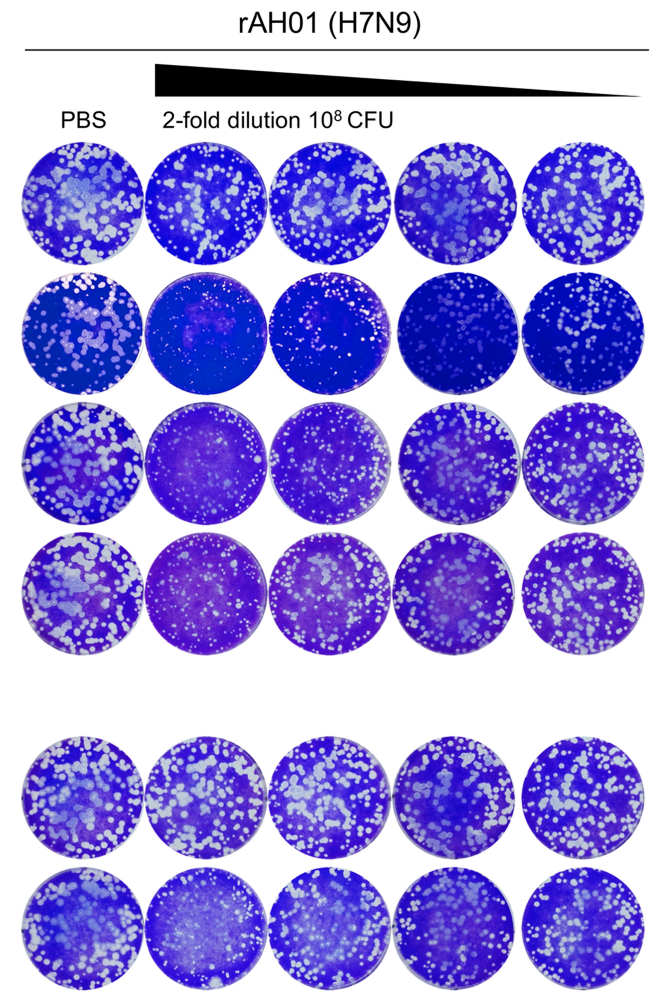

Fig. 2. Plaque-reduction efficacy of Lactobacillus plantarum (Lp) and Leuconostoc mesenteroides (Lm) probiotics against 2009 pandemic influenza H1N1 and avian influenza H7N9 viruses.

The Lp (J1-8, 330, CK10, and 920) and Lm (DRC1506 and 218) strains were evaluated for their plaque-reduction efficacy against rK09 (A) and rAH01 (B) in MDCK cells. Serial 2-fold dilutions of $10^{8} \mathrm{CFU}$ of each probiotic were used in the assay, and PBS was used as a negative control. 


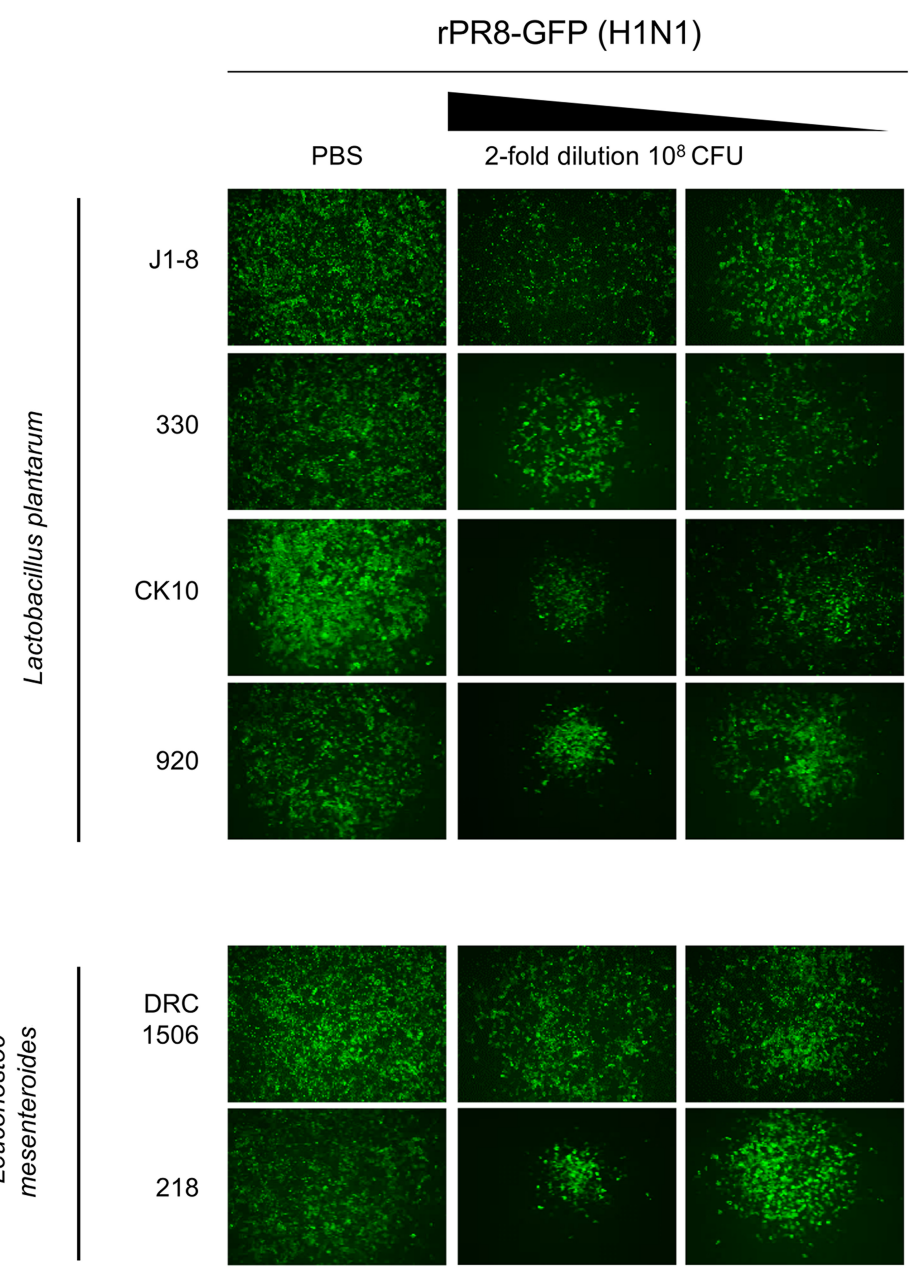

Fig. 3. Inhibition of GFP expression by Lactobacillus plantarum (Lp) and Leuconostoc mesenteroides (Lm) probiotics in GFPexpressing influenza virus-infected cells.

The plaque-reduction efficacy of the Lp (J1-8, 330, CK10, and 920) and Lm (DRC1506 and 218) strains were validated using rPR8-GFP. Under a fluorescence microscope, GFP expression of each viral plaque was examined from low to high magnification at $48-72 \mathrm{hpi}$. The images above were observed with $\times 100$ magnification.

at 3 dpi, mice treated with Lp 330, CK10, and 920 and Lm 218 appeared to be never protected from viral infection because higher viral titers were observed in the lungs of these mice than those of PBS-treated mice. However, at 6 dpi, treatment with Lp 330, CK10, and 920 greatly reduced viral titers with statistical significance $(p<0.05)$ (Fig. 4B). Even though no statistical significance was found, Lm 218 also reduced the viral titer at 6 dpi.

In terms of viral titers in mouse lungs, Lp 330, CK10, and 920 and Lm 218 appeared to be comparable to oseltamivir, which has been prescribed for influenza patients under the name of Tamiflu, because similar ranges of viral titers were determined in the lungs of these mice at $6 \mathrm{dpi}$. In this viral challenge experiment, oseltamivir treatment saved $83.33 \%$ of the mice from death. However, as Lp 330, CK10, and 920 and Lm 218 might not have direct effects against influenza viruses (Fig. 1), treatment with Lp 330, CK10, and 920 and Lm 218 did not protect all the mice from lethal viral challenge. Even so, both Lp CK10 and Lm 218 saved half of

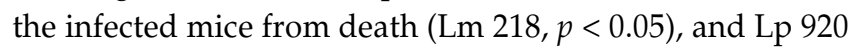
and 330 also resulted in $33.33 \%$ and $16.67 \%$ survival rates,

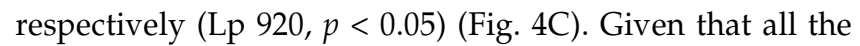
PBS-treated mice had succumbed to death at $8 \mathrm{dpi}$, it is noteworthy that treatment with Lp 330, CK10, and 920 and Lm 218 extended mouse survival from 2.4 to 4.4 days on average (Table 1). These results suggest that despite the 
A

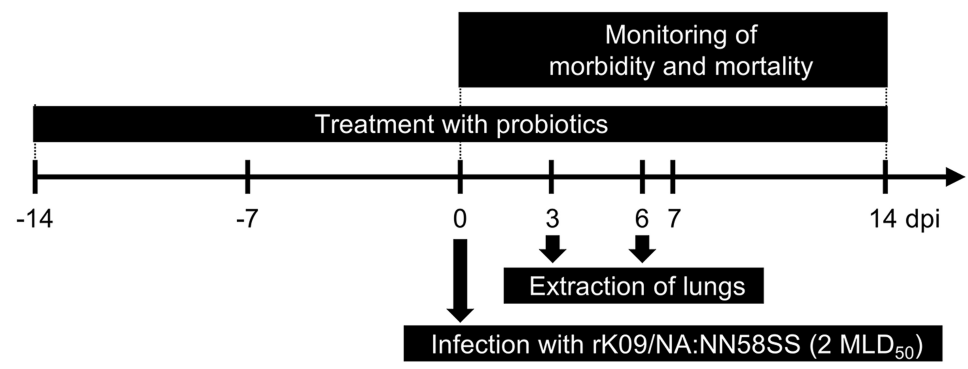

B
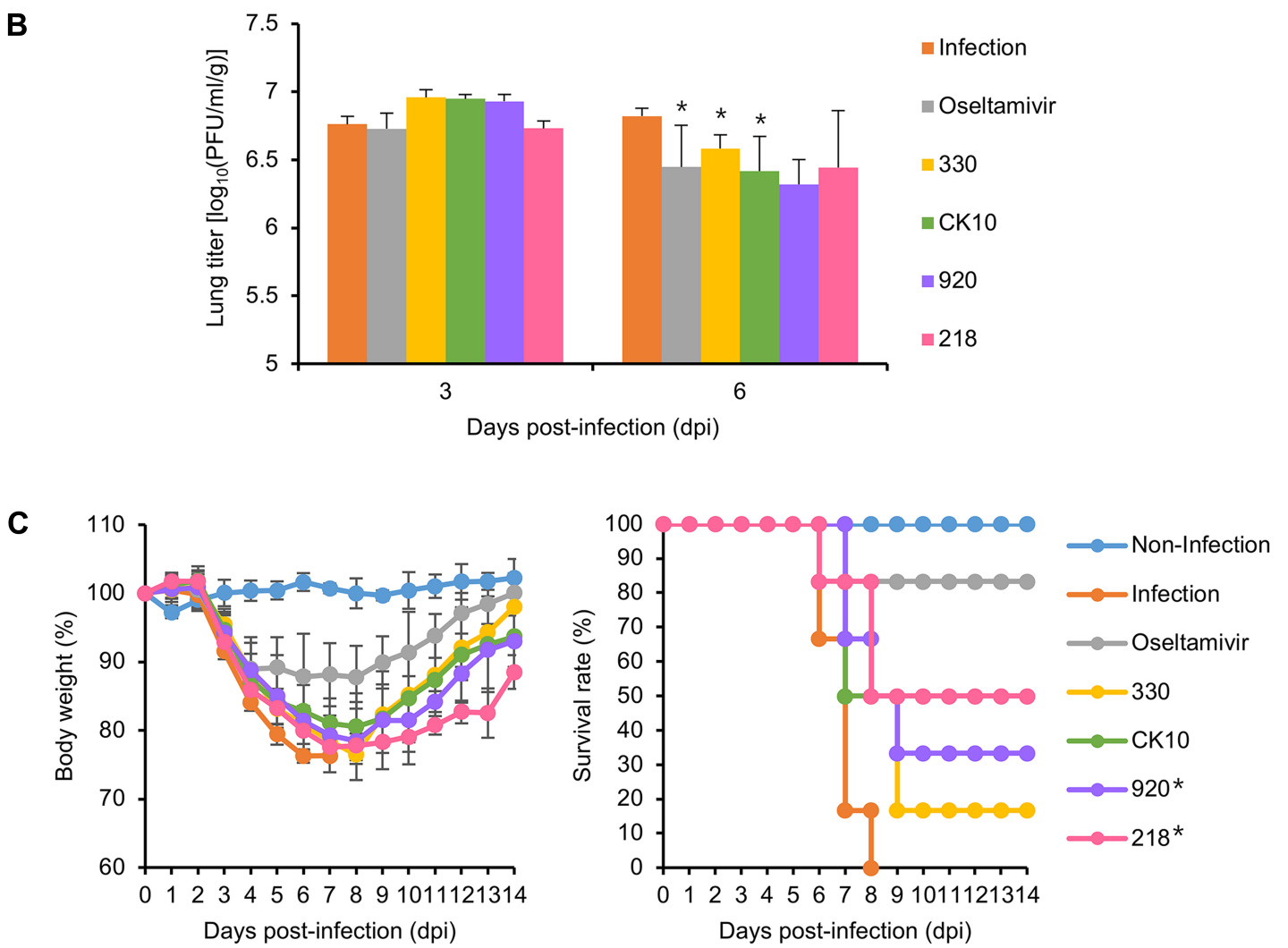

Fig. 4. Effects of Lactobacillus plantarum and Leuconostoc mesenteroides probiotics against lethal viral challenge in mice.

(A) Overall experimental scheme indicating the duration of probiotics administration, infection titer, and time points for infection and lung collection. (B) Viral replication in the lungs of mice was determined by the plaque assay in MDCK cells. PBS was used for mock treatment. The error bar denotes standard deviation (SD). ${ }^{*} p<0.05$. (C) Body weight changes and survival rates of infected mice were monitored daily for $14 \mathrm{dpi}$. PBS was used for the mock infection and treatment. The error bar denotes SD. ${ }^{*} p<0.05$.

rather narrow ranges of protective efficacy, the dietary supplement of Lactobacillus and Leuconostoc probiotics may promote health benefits against influenza

\section{Discussion}

Given the continuous threats of influenza that have been caused mainly by recurrent human seasonal viruses [30] and avian influenza viruses, such as human-infecting H5 and $\mathrm{H7}$ subtypes [31, 32], effective medical arsenals should be prepared for protection not only of human beings but also of animals. Currently, vaccines and antivirals are the two main intervention methods that can be used for prophylactic and therapeutic purposes against human influenza [33, 34]. However, owing to ceaselessly changing patterns of viral genotypes and phenotypes, which may result in the problems of low vaccine effectiveness and antiviral resistance, the quest for reliable new medical 
Table 1. Efficacy of probiotics administration in infected mice.

\begin{tabular}{lcccc}
\hline \multicolumn{1}{c}{ Group } & \% Survival & Body weight loss $(\% \text { mean } \pm \mathrm{SD}(\mathrm{dpi}))^{\mathrm{a}}$ & ${\text { MDD } \pm \mathrm{SD}^{\mathrm{b}}}^{\text {Increase in survival days }^{\mathrm{c}}}$ \\
\hline Non-infection & 100 & - & $14.0 \pm 0$ & 14 \\
Infection & 0 & $23.73 \pm 0.98(6)$ & $5.8 \pm 0.8$ & 0 \\
Oseltamivir & 83.33 & $12.25 \pm 4.58(8)$ & $12.7 \pm 3.3$ & 6.9 \\
330 & 16.67 & $23.56 \pm 1.33(8)$ & $8.2 \pm 3.0$ & 2.4 \\
CK10 & 50 & $19.47 \pm 4.88(8)$ & $9.8 \pm 4.6$ & 4.0 \\
920 & 33.33 & $21.55 \pm 5.69(8)$ & $9.2 \pm 3.8$ & 3.4 \\
218 & 50 & $22.44 \pm 1.59(7)$ & $10.2 \pm 4.3$ & 4.4 \\
\hline
\end{tabular}

${ }^{a}$ The mean maximum body weight losses \pm standard deviations (SD).

${ }^{b} \mathrm{MDD}$, mean number of days to death for the mice that died prior to $14 \mathrm{dpi}$.

${ }^{\mathrm{c}}$ Mean increase in days of mouse survival, compared with mean survival days of the mice of infection groups.

countermeasures have intensified [35-38].

Even though an effective spectrum of probiotics appeared to be substantially variable based on the experimental conditions, such as the kind of virus/probiotic/strain and administration dosage/duration, it was suggested that probiotics supplements may improve vaccine efficacy [39, 40]. Viral pathogenicity might also be reduced with probiotics treatment [41], or certain microbial protein(s) might enhance the efficacy of probiotics treatment [42]. In this regard, dietary supplements of beneficial intestinal microbes as probiotics can be a good option for influenza and influenza-related respiratory patients, especially of the elderly and immunocompromised individuals who might experience the problem of low vaccine effectiveness [43, 44]. Moreover, given the immunomodulatory effects of probiotics [45-47], the administration of probiotics may promote the overall health status of patients. Hence, even though no direct effects of probiotics have been reported on viral pathogenic mechanisms, as demonstrated in our results of virucidal and plaque-reduction assays (Figs. 1-3), daily or regular uptake of probiotics can be suggested as a dietary supplement for targeted population groups. To investigate this, we treated mice with the three Lp and one Lm strains, which were selected on the basis of the results of the plaque-reduction assay (Figs. 2 and 3), 14 days prior to and 14 days after lethal viral challenge. Given the maximal body weight changes of mice, the administration of probiotics appeared to exhibit limited efficacy. However, the Lp- or Lm-treated mice showed maximal body weight loss 1 or 2 days later, compared with the mice of the infection group (Fig. 1C). In addition, by the supplementation with probiotics, the mean days of mouse survival was extended at least 2.4-4.4 days (Table 1). As expected, oseltamivir treatment helped the infected mice to regain their body weights from 6-8 dpi and increased the mean days of survival by approximately 6.9 days (Table 1). Regarding the mechanism of action of oseltamivir, targeting influenza neuraminidase and blocking the release of progeny virions budding out of infected cells [48], it is apparent that oseltamivir might exhibit superior efficacy to the Lp and Lm probiotics. Despite this, the Lp- or Lmtreated mice that experienced approximately $19.47 \%$ to $23.56 \%$ body weight loss started to recover from $8 \mathrm{dpi}$, and 16.67\% (Lp 330), 33.33\% (Lp 920), and 50\% (Lp CK10 and Lm 218) of them survived until 14 dpi (Fig. 4C and Table 1). Consistent with these results, viral replication titers in the lungs of mice were reduced in the Lp- or Lm-treated mice (Fig. 4B).

Here, we do not provide exact evidence of the mechanism(s) of action of the Lp and Lm probiotics against influenza. However, given our results obtained from in vitro and in vivo efficacy analyses, we believe that the tested Lp and Lm probiotics retain beneficial effects against influenza, and our results may underline the usefulness of probiotics as an anti-influenza dietary supplement for overall age groups, including the elderly and immunocompromised patients.

\section{Acknowledgments}

This study was supported by the Korea Institute of Planning and Evaluation for Technology in Food, Agriculture, Forestry and Fisheries (IPET) through High Value-added Food Technology Development Program, funded by the Ministry of Agriculture, Food and Rural Affairs (MAFRA) (Grant No. 314054-3). The funders had no role in the study design, data collection and analysis, decision to publish, or preparation of the manuscript. 


\section{Conflict of Interest}

The authors have no financial conflicts of interest to declare.

\section{References}

1. Palese P. 2004. Influenza: old and new threats. Nat. Med. 10: S82-S87.

2. Neumann G, Kawaoka Y. 2011. The first influenza pandemic of the new millennium. Influenza Other Respir. Viruses 5: 157-166.

3. Webster RG, Govorkova EA. 2014. Continuing challenges in influenza. Ann. N. Y. Acad. Sci. 1323: 115-139.

4. Lam TT, Wang J, Shen Y, Zhou B, Duan L, Cheung CL, et al. 2013. The genesis and source of the H7N9 influenza viruses causing human infections in China. Nature 502: 241-244.

5. Wright PF, Neumann G, Kawaoka Y. 2013. Orthomyxoviruses. In Knipe DM, Howley PM (eds.), Fields Virology, 6th Ed. Ch. 41. Lippincott Williams \& Wilkins, Philadelphia, USA.

6. Krammer F, Garcia-Sastre A, Palese P. 2017. Is it possible to develop a "universal" influenza virus vaccine? Toward a universal influenza virus vaccine: potential target antigens and critical aspects for vaccine development. Cold Spring Harb. Perspect. Biol. 2017: a028845.

7. Hill D, Sugrue I, Arendt E, Hill C, Stanton C, Ross RP. 2017. Recent advances in microbial fermentation for dairy and health. F1000Res. 6: 751.

8. MacDonald TT, Bell I. 2010. Probiotics and the immune response to vaccines. Proc. Nutr. Soc. 69: 442-446.

9. de Vrese M, Winkler P, Rautenberg P, Harder T, Noah C, Laue $\mathrm{C}$, et al. 2006. Probiotic bacteria reduced duration and severity but not the incidence of common cold episodes in a double blind, randomized, controlled trial. Vaccine 24: 6670-6674.

10. Guillemard E, Tondu F, Lacoin F, Schrezenmeir J. 2010. Consumption of a fermented dairy product containing the probiotic Lactobacillus casei DN-114001 reduces the duration of respiratory infections in the elderly in a randomised controlled trial. Br. J. Nutr. 103: 58-68.

11. Boge T, Remigy M, Vaudaine S, Tanguy J, Bourdet-Sicard R, van der Werf S. 2009. A probiotic fermented dairy drink improves antibody response to influenza vaccination in the elderly in two randomised controlled trials. Vaccine 27: 5677-5684.

12. Van Puyenbroeck $K$, Hens $N$, Coenen $S$, Michiels $B$, Beunckens C, Molenberghs G, et al. 2012. Efficacy of daily intake of Lactobacillus casei Shirota on respiratory symptoms and influenza vaccination immune response: a randomized, double-blind, placebo-controlled trial in healthy elderly nursing home residents. Am. J. Clin. Nutr. 95: 1165-1171.

13. Pessione E, Cirrincione S. 2016. Bioactive molecules released in food by lactic acid bacteria: encrypted peptides and biogenic amines. Front. Microbiol. 7: 876.

14. Cho J, Lee D, Yang C, Jeon J, Kim J, Han H. 2006. Microbial population dynamics of kimchi, a fermented cabbage product. FEMS Microbiol. Lett. 257: 262-267.

15. Jung JY, Lee SH, Jeon CO. 2014. Kimchi microflora: history, current status, and perspectives for industrial kimchi production. Appl. Microbiol. Biotechnol. 98: 2385-2393.

16. Choi CY, Kim YH, Oh S, Lee HJ, Kim JH, Park SH, et al. 2017. Anti-inflammatory potential of a heat-killed Lactobacillus strain isolated from kimchi on house dust mite-induced atopic dermatitis in NC/Nga mice. J. Appl. Microbiol. 123: 535-543.

17. Park S, Kim JI, Bae JY, Yoo K, Kim H, Kim IH, et al. 2018. Effects of heat-killed Lactobacillus plantarum against influenza viruses in mice. J. Microbiol. 56: 145-149.

18. Lee YD, Hong YF, Jeon B, Jung BJ, Chung DK, Kim H. 2016. Differential cytokine regulatory effect of three Lactobacillus strains isolated from fermented foods. J. Microbiol. Biotechnol. 26: 1517-1526.

19. Kwak MK, Liu R, Kwon JO, Kim MK, Kim AH, Kang SO. 2013. Cyclic dipeptides from lactic acid bacteria inhibit proliferation of the influenza A virus. J. Microbiol. 51: 836-843.

20. Maeda N, Nakamura R, Hirose Y, Murosaki S, Yamamoto Y, Kase $\mathrm{T}$, et al. 2009. Oral administration of heat-killed Lactobacillus plantarum L-137 enhances protection against influenza virus infection by stimulation of type I interferon production in mice. Int. Immunopharmacol. 9: 1122-1125.

21. Kiso M, Takano R, Sakabe S, Katsura H, Shinya K, Uraki R, et al. 2013. Protective efficacy of orally administered, heatkilled Lactobacillus pentosus b240 against influenza A virus. Sci. Rep. 3: 1563.

22. Goto H, Sagitani A, Ashida N, Kato S, Hirota T, Shinoda T, et al. 2013. Anti-influenza virus effects of both live and nonlive Lactobacillus acidophilus L-92 accompanied by the activation of innate immunity. Br. J. Nutr. 110: 1810-1818.

23. Kim JI, Lee I, Park S, Park MS. 2012. Surface glycoproteins determine the feature of the 2009 pandemic H1N1 virus. BMB Rep. 45: 653-658.

24. Kim JI, Lee I, Park S, Hwang MW, Bae JY, Lee S, et al. 2013. Genetic requirement for hemagglutinin glycosylation and its implications for influenza A H1N1 virus evolution. J. Virol. 87: 7539-7549.

25. Park S, Kim JI, Lee I, Bae JY, Yoo K, Nam M, et al. 2017. Adaptive mutations of neuraminidase stalk truncation and deglycosylation confer enhanced pathogenicity of influenza A viruses. Sci. Rep. 7: 10928.

26. Manicassamy B, Manicassamy S, Belicha-Villanueva A, Pisanelli G, Pulendran B, Garcia-Sastre A. 2010. Analysis of in vivo dynamics of influenza virus infection in mice using a GFP reporter virus. Proc. Natl. Acad. Sci. USA 107: 11531-11536.

27. Kim JI, Park S, Lee I, Lee S, Shin S, Won Y, et al. 2012. GFPexpressing influenza A virus for evaluation of the efficacy of antiviral agents. J. Microbiol. 50: 359-362. 
28. Lee I, Kim JI, Park S, Bae JY, Yoo K, Yun SH, et al. 2017. Single PA mutation as a high yield determinant of avian influenza vaccines. Sci. Rep. 7: 40675.

29. Park S, Kim JI, Lee I, Lee S, Hwang MW, Bae JY, et al. 2013. Aronia melanocarpa and its components demonstrate antiviral activity against influenza viruses. Biochem. Biophys. Res. Commun. 440: 14-19.

30. Centers for Disease Control and Prevention (CDC). 2018. Disease burden of influenza. Available at https://www. cdc.gov/flu/about/disease/burden.htm.Accessed March 16, 2018

31. World Health Organization (WHO). 2018. Cumulative number of confirmed human cases of avian influenza A(H5N1) reported to WHO. Available at http://www.who.int/influenza/ human_animal_interface/H5N1_cumulative_table_archives/ en/. Accessed March 2, 2018.

32. Food and Agriculture Organization of the United Nations (FAO). 2018. H7N9 situation. Available at http://www.fao. org/ag/againfo/programmes/en/empres/H7N9/situation_ update.html). Accessed March 28, 2018.

33. Fiore AE, Fry A, Shay D, Gubareva L, Bresee JS, Uyeki TM, et al. 2011. Antiviral agents for the treatment and chemoprophylaxis of influenza - recommendations of the Advisory Committee on Immunization Practices (ACIP). MMWR Recomm. Rep. 60: 1-24.

34. Centers for Disease Control and Prevention (CDC). 2018. Seasonal influenza vaccine effectiveness, 2005-2018. Available at https://www.cdc.gov/flu/professionals/vaccination/ effectiveness-studies.htm). Accessed March 15, 2018.

35. Nachbagauer R, Krammer F. 2017. Universal influenza virus vaccines and therapeutic antibodies. Clin. Microbiol. Infect. 23: 222-228.

36. Harding AT, Heaton NS. 2018. Efforts to improve the seasonal influenza vaccine. Vaccines (Basel) 6: E19.

37. Naesens L, Stevaert A, Vanderlinden E. 2016. Antiviral therapies on the horizon for influenza. Curr. Opin. Pharmacol. 30: 106-115.

38. Park S, Kim JI, Park MS. 2012. Antiviral agents against influenza viruses. J. Bacteriol. Virol. 42: 284-293.
39. Zimmermann P, Curtis N. 2018. The influence of probiotics on vaccine responses - a systematic review. Vaccine 36: 207-213.

40. Vitetta L, Saltzman ET, Thomsen M, Nikov T, Hall S. 2017. Adjuvant probiotics and the intestinal microbiome: enhancing vaccines and immunotherapy outcomes. Vaccines (Basel) 5: 50.

41. Chen MF, Weng KF, Huang SY, Liu YC, Tseng SN, Ojcius DM, et al. 2017. Pretreatment with a heat-killed probiotic modulates monocyte chemoattractant protein- 1 and reduces the pathogenicity of influenza and enterovirus 71 infections. Mucosal Immunol. 10: 215-227.

42. Starosila D, Rybalko S, Varbanetz L, Ivanskaya N, Sorokulova I. 2017. Anti-influenza activity of a Bacillus subtilis probiotic strain. Antimicrob. Agents Chemother. 61: e00539-17.

43. Demicheli V, Jefferson T, Di Pietrantonj C, Ferroni E, Thorning S, Thomas RE, et al. 2018. Vaccines for preventing influenza in the elderly. Cochrane Database Syst. Rev. 2: CD004876.

44. Bitterman R, Eliakim-Raz N, Vinograd I, Zalmanovici Trestioreanu A, Leibovici L, Paul M. 2018. Influenza vaccines in immunosuppressed adults with cancer. Cochrane Database Syst. Rev. 2: CD008983.

45. Yeh TL, Shih PC, Liu SJ, Lin CH, Liu JM, Lei WT, et al. 2018. The influence of prebiotic or probiotic supplementation on antibody titers after influenza vaccination: a systematic review and meta-analysis of randomized controlled trials. Drug Des Devel. Ther. 12: 217-230.

46. Jung YJ, Lee YT, Ngo VL, Cho YH, Ko EJ, Hong SM, et al. 2017. Heat-killed Lactobacillus casei confers broad protection against influenza A virus primary infection and develops heterosubtypic immunity against future secondary infection. Sci. Rep. 7: 17360.

47. Lei WT, Shih PC, Liu SJ, Lin CY, Yeh TL. 2017. Effect of probiotics and prebiotics on immune response to influenza vaccination in adults: a systematic review and meta-analysis of randomized controlled trials. Nutrients 9: 1175.

48. Palese P, Tobita K, Ueda M, Compans RW. 1974. Characterization of temperature sensitive influenza virus mutants defective in neuraminidase. Virology 61: 397-410. 